\title{
The origin of CEMA and its relation to CSP
}

\author{
Dimitris A. Goussis ${ }^{\mathrm{a}, *}$, Hong G. Im ${ }^{\mathrm{b}}$, Habib N. Najm ${ }^{\mathrm{c}}$, Samuel Paolucci ${ }^{\mathrm{d}}$, Mauro Valorani ${ }^{\mathrm{e}}$ \\ ${ }^{a}$ Department of Mechanical Engineering, Khalifa University of Science, Technology and Research, Abu \\ Dhabi 127788, United Arab Emirates \\ ${ }^{b}$ Clean Combustion Research Center (CCRC), King Abdullah University of Science and Technology \\ (KAUST), Thuwal, 23955-6900, Saudi Arabia \\ ${ }^{c}$ Combustion Research Facility, Sandia National Laboratories, Livermore, CA 94551, United States \\ ${ }^{d}$ Aerospace and Mechanical Engineering Department, University of Notre Dame, Notre Dame, IN, United \\ States \\ ${ }^{e}$ Mechanical and Aerospace Engineering Department, Sapienza University, Rome 00184, Italy
}

\begin{abstract}
There currently exist two methods for analysing an explosive mode introduced by chemical kinetics in a reacting process: the Computational Singular Perturbation (CSP) algorithm and the Chemical Explosive Mode Analysis (CEMA). CSP was introduced in 1989 and addressed both dissipative and explosive modes encountered in the multi-scale dynamics that characterize the process, while CEMA was introduced in 2009 and addressed only the explosive modes. It is shown that (i) the algorithmic tools incorporated in CEMA were developed previously on the basis of CSP and (ii) the examination of explosive modes has been the subject of CSP-based works, reported before the introduction of CEMA.
\end{abstract}

Keywords: chemical kinetics, multi-scale analysis, CSP, CEMA

\section{Introduction}

The realization of the multi-scale character of the dynamics that develop in combustion processes, and the use of appropriate analysis methodologies, lead to significant findings. Among them are the hydrodynamic limit (small flame thickness), small Mach number asymptotics, Damköhler number asymptotics and activation energy asymptotics; see review papers [1-3]. The pioneering work of Peters, Kee, Williams and Jones extended the multiscale analysis in the field of reduced chemical kinetic modeling, by exploiting the fast dynamics and by implementing the quasi-steady state and partial equilibrium approximations [4-7]. This work allowed for the identification of (i) the constraints in which the reacting process is bound to evolve (in the form of steady state or partial equilibrium relations) and (ii) the reduced model that governs the evolution within the established constraints. The dominant dynamics of the reduced model could be of either dissipative or explosive nature; i.e., dynamics that lead the system towards or away from equilibrium, respectively [8].

The construction of large chemical kinetic mechanisms necessitated the development of algorithmic methodologies for multi-scale analysis; see Refs. [9-15] for a review of these methods.

\footnotetext{
${ }^{*}$ Corresponding author: dimitris.goussis@ku.ac.ae
} 
One of these methodologies is Computational Singular Perturbation (CSP), which was introduced in 1989 as "The basic idea of CSP is that the large number of physically meaningful elementary reactions in a complex reaction system can be grouped into separate reaction groups, each identified with a single characteristic time scale" [8]. CSP has been employed for the development of a solver for stiff ODEs, the construction of reduced and skeletal mechanisms, and the acquisition of all relevant physical understanding; e.g. [16-22]. The usefulness of the method in analyzing multi-scale systems was examined and it was concluded that it can generate, order by order, the results of asymptotic analysis [23-26]. Concepts introduced by CSP were later employed for the development of other multi-scale algorithmic methodologies for the analysis of combustion processes, which all introduced new concepts and algorithmic tools; i.e., ILDM [27, 28], G-Scheme [29, 30] and TSR [31, 32].

The Chemical Explosive Mode Analysis (CEMA) was first introduced in 2009 in two conference papers [33, 34]. Regarding journal papers, CEMA was first introduced in the 2010 Combustion and Flame paper by Liu et al. [35] and later in the same year in the Journal of Fluid Mechanics paper by Lu et al [36]. Since its introduction, CEMA has been employed for the analysis of a large number of combustion processes, including autoignition, flames and turbulent reacting flows; e.g., [37-43].

CSP examines the explosive mode in the context of multi-scale (fast/slow) dynamics, as one among the slow modes. Therefore, its influence is frequently assessed (i) by examining the proximity of the explosive time scale to the fastest time scales of the slow modes and (ii) by comparing the amplitude of the explosive mode with the amplitude of all slow ones [4446]. In addition, the conclusions reported are validated by perturbing the pre-exponential factor of the reactions deemed responsible for the development of the explosive time scale $[44,46,47]$. In contrast, the multi-scale character of the governing equations is not discussed in the CEMA literature. Therefore, CEMA does not allow for the possibility that an explosive mode might not influence the evolution of the reacting process, promoting thus the notion that the action of an explosive mode is significant under all circumstances. This notion is advocated by a number of statements distancing CEMA from CSP. For example, although CSP is based on the chemical source term and was introduced by analyzing homogeneous mixtures [8, 48, 49], Ref. [33] states that "Compared to the classical CSP theory based on the full Jacobian matrix, the present method utilizes the Jacobian matrix of the chemical source term and is therefore simple to implement, even for many complex flow fields" and Ref. [34] states that "Compared with CSP modes, which may be affected by transport, CEM was defined on the chemical Jacobian, such that CEM is purely a chemical property. Therefore major differences exist in the application of CEMA and CSP modes". Finally, Ref. [36], states that"It is, however, important to emphasize at the outset of this study that, while some concepts from CSP were adopted in the formulation of CEMA, this new formulation is mathematically and fundamentally distinctive from CSP." However, the fact is that reacting processes are multi-scale in character. In contrast to CSP, CEMA overlooks altogether this feature and focuses on the possible existence of an explosive mode, without considering its relation to the other modes [44-46, 50, 51].

The explosive mode was first introduced in the combustion community in 1989, in the context of a homogeneous autoignition of a $\mathrm{H}_{2} / \mathrm{O}_{2}$ mixture study, reported in the first CSP paper [8]. A more detailed study of an explosive mode was published in 1997, in the study of ignition of counter-flowing methane versus heated air [52]. The analysis was based on 
CSP and it was concluded that "CSP provides an ordering of the elementary reactions within the explosive mode, based on their respective "participation index", a measure of the contribution from individual reactions to the amplitude of the mode". CSP was employed in 2005 for the investigation of the explosive modes developing in homogeneous ignition and premixed flames [53]. The 2006 paper titled "Computational Singular Perturbation Analysis of Two-Stage Ignition of Large Hydrocarbons" examined the explosive mode in the dynamics of a homogeneous n-heptane/air autoignition [54]. Similar CSP-based investigations were reported in 2007 and 2008 for the analysis of the explosive mode in the dynamics of a model exploring the combustion enhancement by DME addition to methane/air mixtures and the DME autoignition at elevated pressures, respectively $[55,56]$. In 2006 a mathematical model of the circadian cycle was investigated with CSP, by exploring the development of an explosive mode in parts of the cycle [57]. Another study of the explosive mode was reported in the 2008 paper titled "Analysis of a turbulent lifted hydrogen/air jet flame from direct numerical simulation with Computational Singular Perturbation" [58].

Following the introduction of CEMA in 2009, the role of the explosive mode was the subject of investigations, in which the method employed was referred as either CSP $[22,59-$ 66] or CEMA [37-43, 67, 68]. Naturally, the co-existence of the two acronyms CSP and CEMA for the same method generated confusion. For example, there are papers claiming that exactly the same methodology is CSP-based in 1997 [52] and CEMA-based in 2010 [35]. In addition, there are papers claiming to use CEMA, but in fact they cite CSP as its origin [35, 41]; i.e., they cite the paper titled "Analysis of a Turbulent Lifted Hydrogen/Air Jet Flame from Direct Numerical Simulation with CSP" [58]. Moreover, in the paper [67] the CSP-based algorithmic tool "Importance Index" [20, 48] is correctly referred as a CSP tool, but the "Participation Index" $[18,69]$ is referred as a CEMA tool. Finally, CSP-based papers are characterized as CEMA-based ones [68].

It is the aim of this paper to resolve this confusion by comparing the algorithmic tools incorporated in CEMA with those developed previously by CSP. First, the CSP tools will be briefly presented and the cases in which these tools were employed for the analysis of explosive dynamics before the introduction of CEMA will be stated. Then, the CEMA tools will be presented and their relation to the CSP ones will be established. The present work aims at clarifying the relation of CSP and CEMA, since both are employed for the analysis of explosive modes that develop in reacting processes.

\section{The CSP-based diagnostic tools and their use for the analysis of dissipative and explosive modes}

Consider for simplicity the governing equations for adiabatic autoignition of a homogeneous fuel/air mixture at constant volume:

$$
\frac{d \mathbf{y}}{d t}=\mathbf{g}(\mathbf{y})=\sum_{k=1}^{2 K} \mathbf{S}_{k}(\mathbf{y}) R^{k}(\mathbf{y})
$$

where the $(N+1)$-dim. state vector $\mathbf{y}=\left[y^{1}, y^{2}, \cdots, y^{N}, T\right]$ incorporates $N$ mass fractions and temperature, while the vector field $\mathbf{g}(\mathbf{y})$ can be expressed as a sum of $2 K$ products $\mathbf{S}_{k}(\mathbf{y}) R^{k}(\mathbf{y})$ of the generalized stoichiometric vector of the $k$-th reaction and its reaction 
rate of progress; forward and backward directions are considered as separate reactions. According to CSP, the vector field $\mathbf{g}(\mathbf{y})$ is resolved in $N+1$ modes, each one of which is characterized by a distinct time scale, say $\tau_{n}$ [8]. Thus, Eq. (1) can be cast in the form $[8,70]$ :

$$
\frac{d \mathbf{y}}{d t}=\mathbf{g}(\mathbf{y})=\sum_{n=1}^{N+1} \mathbf{a}_{n}(\mathbf{y}) f^{n}(\mathbf{y}) \quad f^{n}(\mathbf{y})=\mathbf{b}^{n}(\mathbf{y}) \cdot \mathbf{g}(\mathbf{y})=\sum_{k=1}^{2 K}\left(\mathbf{b}^{n}(\mathbf{y}) \cdot \mathbf{S}_{k}(\mathbf{y})\right) R^{k}(\mathbf{y})
$$

where $\mathbf{a}_{n}$ is the $(N+1)$-dim. CSP column basis vector of the $n$-th mode, $\mathbf{b}^{n}$ is the $(N+1)$ dim. row vector $\left(\mathbf{b}^{i} \cdot \mathbf{a}_{j}=\delta_{j}^{i}\right)$ and $f^{n}$ is the related amplitude [8, 49]. When the dynamics of the system in Eq. (2) exhibit $M$ time scales that are (i) of dissipative nature (i.e. the components of the system that generate them tend to drive the system towards a fixed point) and (ii) much faster than the rest, then Eq. (2) simplifies to:

$$
f^{r}(\mathbf{y}) \approx 0 \quad(r=1, \ldots, M) \quad \frac{d \mathbf{y}}{d t} \approx \sum_{s=M+1}^{N+1} \mathbf{a}_{s}(\mathbf{y}) f^{s}(\mathbf{y})
$$

when these $M$ fast time scales become exhausted. In essence, Eq. (3) states that the evolution of the system depends only on the slow modes. The first relation in Eq. (3) is an $M$-dim. system of algebraic equations and defines the manifold $\mathscr{M}$ (a low dimensional surface in phase-space, where the system is confined to evolve), while the second relation is an $(N+1)$-dim. system of ODEs that governs the slow evolution of the system on this manifold.

The basis vectors $\mathbf{a}_{n}(\mathbf{y})$ and $\mathbf{b}^{n}(\mathbf{y})$ are approximated by a pair of refinement procedures, which involve the Jacobian $\mathbf{J}$ of the chemical kinetic term $\mathbf{g}$; one refinement providing higher order accuracy and the other stability of the simplified model in Eq. (3) [8, 71]. For leading order accuracy, the CSP vectors can be approximated by the right and left eigenvectors of $\mathbf{J}$; i.e., $\mathbf{a}_{i}=\boldsymbol{\alpha}_{i}$ and $\mathbf{b}^{i}=\boldsymbol{\beta}^{i}$, where $\boldsymbol{\alpha}_{i}$ and $\boldsymbol{\beta}^{i}$ are the right and left eigenvectors of $\mathbf{J}$, respectively [8, 72]. Eigenvectors were employed as CSP vectors in numerous works from 1989 and on; e.g., [18, 19, 21, 23, 70, 73].

Although CSP was initially developed for the analysis of spatially homogeneous systems, its use was later extended for the analysis of non-homogenous ones [71, 74] of the form:

$$
\frac{d \mathbf{y}}{d t}=\mathbf{L}(\mathbf{y})+\mathbf{g}(\mathbf{y})
$$

where the $\mathbf{L}(\mathbf{y})$ represents transport and $\mathbf{g}(\mathbf{y})$ represents chemical kinetics, as in Eq. (1). In this case, the CSP form of the governing equations and the reduced model is of the form of Eqs. (2) and (3), with the amplitudes now defined as:

$$
f^{n}(\mathbf{y})=\mathbf{b}^{n}(\mathbf{y}) \cdot[\mathbf{L}(\mathbf{y})+\mathbf{g}(\mathbf{y})]
$$

The CSP basis vectors in this spatially inhomogeneous case are computed with the same methodology as in the homogeneous one. However, the presence of the transport term allows for leading order accurate basis vectors only; i.e., vectors approximated by one pair of refinements or by the eigenvectors of $\mathbf{J}[21,22,71,75]$. 
The availability of the CSP basis vectors led to the development of a number of algorithmic diagnostic tools; among them are (i) the time scale, (ii) the CSP Pointer, (iii) the Participation Index and (iv) the Time scale Participation Index.

The time scale of the $i$-th mode was introduced in the 1989 Symposium paper [8] as the reciprocal of the modulus of the $i$-th eigenvalue $\lambda_{i}$ of the Jacobian $\mathbf{J}$; see also [16]. These time scales were distinguished as dissipative (relaxation) or explosive, depending on whether the real part of the related eigenvalue is negative or positive; i.e., depending on whether the related mode is leading the trajectory towards a fixed point or away from it. It was recognized that a positive eigenvalue characterizes an explosive mode, which by definition is one of the slow ones [8,72]. In particular, by considering homogeneous ignition of a simple $\mathrm{H}_{2} / \mathrm{O}_{2}$ system, it was shown that a positive eigenvalue might develop at the early stages of the process $[8,72]$. Since then, explosive time scales have been examined in numerous works; e.g., $[22,54-56,60,76,77]$.

The CSP Pointer (or Radical Pointer) was introduced in the 1992 Symposium paper [48] as an algorithmic tool for the identification of variables related the most to the fast modes; i.e., the variables that will respond the most to a perturbation along the fast directions [21]. The physical significance of this tool was examined in a later study, where it was shown that the variables identified by the Pointer of the $i$-th mode are those that are characterized by the related time scale $\tau_{i}$ and participate in the expressions of the rates $R^{k}$ that contribute significantly to the expression of the related $i$-th fast amplitude $f^{i} \approx 0$ [21]. The use of the Pointer was then extended in the 2006 SIAM paper [57] to the slow modes, characterized by either dissipative or explosive dynamics. Considering the $i$-th mode, the CSP Pointer is defined by the expression:

$$
\mathbf{D}^{i}=\operatorname{diag}\left[\mathbf{a}_{i} \mathbf{b}^{i}\right]=\left[a_{i}^{1} b_{1}^{i}, a_{i}^{2} b_{2}^{i}, \ldots, a_{i}^{N+1} b_{N+1}^{i}\right]
$$

where, due to the orthogonality condition $\mathbf{b}^{i} \cdot \mathbf{a}_{j}=\delta_{j}^{i}$, the sum of all $N+1$ elements of $\mathbf{D}^{i}$ equals unity $[48,49]$. A large value of $a_{i}^{n} b_{n}^{i}$ indicates a strong association of the $n$-th variable with the $i$-th mode.

The Participation Index (PI) was introduced in the 1990 AIAA paper [69] and in a simpler form in the 1999 CST paper [18], as an algorithmic tool to assess the contribution of the various reaction rates to the occurring cancellations in the expression of the exhausted fast amplitudes $f^{i} \approx 0$. The use of the PI was later extended for the analysis of slow amplitudes; in particular, for the first time for explosive modes in the $1997 \mathrm{C} \& \mathrm{~F}$ paper [52], followed by the 2006 JPCA paper [54], the 2007 Symposium paper [55] and the 2011 C\&F paper [22]. Considering the $i$-th mode, the PI is defined by the expression:

$$
P_{k}^{i}=\frac{\left(\mathbf{b}^{i} \cdot \mathbf{S}_{k}\right) R^{k}}{\sum_{j=1}^{2 K}\left|\left(\mathbf{b}^{i} \cdot \mathbf{S}_{j}\right) R^{j}\right|} \quad k=1, \ldots, 2 K
$$

where by definition $\sum_{k=1}^{2 K}\left|P_{k}^{r}\right|=1[18,78]$. A large value of $P_{k}^{i}$ indicates a significant contribution of the $k$-th reaction to the $i$-th mode amplitude.

Finally, the Time scale Participation Index (TPI) was introduced in the 2006 SIAM paper [57], as an algorithmic tool to assess the contribution of the various reactions to the development of the time scale $\tau_{i}=\left|\lambda_{i}\right|^{-1}$. The TPI was used there in order to analyze fast 
and slow time scales, of either dissipative or explosive character. Considering the $i$-th mode, the TPI is defined by the expression:

$$
J_{k}^{i}=\frac{\mathbf{b}^{i} \mathbf{J}_{k} \mathbf{a}_{i}}{\sum_{j=1}^{2 K}\left|\mathbf{b}^{i} \mathbf{J}_{j} \mathbf{a}_{i}\right|} \quad k=1, \ldots, 2 K
$$

where $\mathbf{b}^{i}$ and $\mathbf{a}_{i}$ are the left and right eigenvectors, respectively, of the Jacobian $\mathbf{J}$ that relate to $\lambda_{i}=\mathbf{b}^{i} \mathbf{J}_{1} \mathbf{a}_{i}+\cdots+\mathbf{b}^{i} \mathbf{J}_{2 K} \mathbf{a}_{i}, \mathbf{J}_{k}$ is the contribution of the $k$-th reaction to the Jacobian $\mathbf{J}=\mathbf{J}_{1}+\cdots+\mathbf{J}_{2 K}$ and by definition $\sum_{k=1}^{2 K}\left|J_{k}^{i}\right|=1$. A large positive or negative value of $J_{k}^{i}$ indicates a significant contribution of the $k$-th reaction towards the development of an explosive or dissipative, respectively, $i$-th time scale.

The PI and CSP Pointer were first introduced in 1990 and 1992, respectively, by considering real basis vectors. In the 2006 SIAM paper [57], where the TPI was introduced and the CSP vectors were approximated by the eigenvectors of $\mathbf{J}$, the expressions in Eqs. (6)-(8) were extended to the case of complex basis vectors.

\section{The CEMA tools and their use for the analysis of explosive modes}

Of all time scales in the dynamics of the model, CEMA concentrates on the explosive time scale, which by definition is accounted among the slow ones [8]. Progressively, CEMA incorporated notions and tools introduced by CSP, as follows.

In the two 2009 conference papers [33, 34] and the $2010 \mathrm{C} \& \mathrm{~F}$ and JFM papers [35, 36] the explosive time scale and the CSP Pointer were assigned to CEMA. Regarding the explosive time scale, it was stated "The eigenvalues therefore indicate the reciprocal characteristic time scales of the modes. A positive eigenvalue of the chemical Jacobian indicates a chemical explosive mode, which is a chemical property of the mixture" [36]. A similar concept was introduced in the 1989 Symposium paper [8], in which the explosive time scale as the reciprocal of a positive eigenvalue was first introduced in the model reduction literature, along with the term "explosive mode".

In addition, the "Explosion Pointer" was introduced in [36] as:

$$
\mathbf{E P}=\operatorname{diag}\left[\mathbf{a}_{\exp } \mathbf{b}^{\exp }\right]=\left[a_{\text {exp }}^{1} b_{1}^{e x p}, a_{\text {exp }}^{2} b_{2}^{e x p}, \ldots, a_{\text {exp }}^{N+1} b_{N+1}^{e x p}\right]
$$

where $\mathbf{a}_{\exp }$ and $\mathbf{b}^{\exp }$ are the right and left eigenvectors of $\mathbf{J}$ that relate to the explosive mode. The similarity of EP with the CSP (Radical) Pointer in Eq. (6) is obvious. This is acknowledged in [36], but it is stated that "To avoid confusion with the radical pointer, EP will be referred to as the 'explosion pointer' in the following". In addition, the "Explosion Index" was introduced in the same paper as:

$$
\mathbf{E I}=\frac{|\mathbf{E P}|}{\sum_{n=1}^{N+1}\left|E P_{n}\right|}=\frac{1}{\sum_{n=1}^{N+1}\left|a_{\text {exp }}^{n} b_{n}^{\exp }\right|}\left[\left|a_{\text {exp }}^{1} b_{1}^{\exp }\right|,\left|a_{\text {exp }}^{2} b_{2}^{\exp }\right|, \ldots,\left|a_{\text {exp }}^{N+1} b_{N+1}^{\exp }\right|\right]
$$

which is a scaled EP; i.e., a scaled CSP Pointer. Both EP and EI were employed for the identification of the variable related the most to the explosive mode; i.e., the same use as the CSP Pointer, which addressed both explosive and dissipative modes. 
In the $2010 \mathrm{C} \& \mathrm{~F}$ paper [35] a "Participation Index" for the explosive mode is employed with the statement "An explosion participation index, associated with the chemical explosive mode, is defined analogous to the concept of the participation index in the theory of CSP." However, no mathematical expression for this index is provided, but only a figure displaying positive and negative indices. A mathematical formula for the "Participation Index" PI is provided in the $2012 \mathrm{C} \& \mathrm{~F}$ paper [38] along with a new expression for the "Explosion Index" EI as:

$$
\mathbf{E I}=\frac{\left|\mathbf{a}_{\text {exp }} \otimes \mathbf{b}^{\exp }\right|}{s u m\left|\mathbf{a}_{\text {exp }} \mathbf{b}^{\text {exp }}\right|} \quad \mathbf{P I}=\frac{\left|\left(\mathbf{b}^{\exp } \cdot \mathbf{S}\right) \otimes \mathbf{R}\right|}{s u m\left|\left(\mathbf{b}^{\exp } \cdot \mathbf{S}\right) \otimes \mathbf{R}\right|}
$$

where $\mathbf{S}=\left[\mathbf{S}_{1}, \mathbf{S}_{2}, \cdots, \mathbf{S}_{K}\right]$ is the stoichiometric matrix, $\mathbf{R}=\left[R^{\text {net }, 1}, R^{\text {net }, 2}, \cdots, R^{\text {net }, K}\right]$ is the vector of the net reaction rates and the symbol $\otimes$ denotes element-wise product of two vectors. It is stated in [38] that "The definitions of $\boldsymbol{E I}$ and $\boldsymbol{P I}$ are similar to the radical pointer and the participation index, respectively, in the CSP theory". Indeed, the expression of EI in Eq. (11) is similar to that in Eq. (9), which was shown to be a scaled CSP Pointer. Regarding PI, its elements are:

$$
P I_{k}=\frac{\left|\left(\mathbf{b}^{e x p} \cdot \mathbf{S}_{k}\right) R^{n e t, k}\right|}{\sum_{i=1}^{K}\left|\left(\mathbf{b}^{e x p} \cdot \mathbf{S}_{k}\right) R^{n e t, k}\right|} \quad k=1, \ldots, K
$$

i.e., now the indices are by definition all positive, in contrast with the results displayed in [35]. Later on, the absolute value in the numerator was removed, so that $P I_{k}$ could be positive or negative [42], as with the equivalent CSP quantity $P_{k}^{i}$ in Eq. (7). According to [38], $P I_{k}$ was introduced in order to assess the contribution of the $k$-th reaction to the amplitude of an explosive mode; i.e., the same use as the CSP index PI, which addressed both explosive and dissipative modes, as discussed in the previous section. The similarity of the "Participation Index" $P I_{k}$ in Eq. (12) to the CSP index $P_{k}^{i}$ in Eq. (7) is obvious. The only difference is that in $P I_{k}$ the contribution of the net rate of each reaction is considered, while in $P_{k}^{i}$ the contributions of the forward and backward directions of each reaction are considered separately.

In the $2014 \mathrm{C} \& \mathrm{~F}$ paper [40], the "Bifurcation Index" $\mathbf{B I}^{k}$ was introduced for the analysis of a homogeneous model involving $K+1$ processes ( $K$ reactions and mixing) as:

$$
B I^{k}=\frac{\operatorname{Re}\left(\lambda_{k}^{*}\right)}{\max _{k=1, K}\left|\operatorname{Re}\left(\lambda_{k}^{*}\right)\right|} \quad k=1, \ldots, K+1
$$

where $\operatorname{Re}(\cdot)$ denotes the real part of a complex number and $\operatorname{Re}\left(\lambda_{k}^{*}\right)$ denotes the contribution of the $k$-th process to the real part of the eigenvalue $\lambda^{*}$ of the Jacobian $\mathbf{J}$, which involves both kinetics and mixing:

$$
\operatorname{Re}\left(\lambda^{*}\right)=\operatorname{Re}\left(\mathbf{b}^{*} \mathbf{J} \mathbf{a}_{*}\right)=\sum_{k=1}^{K+1} \operatorname{Re}\left(\mathbf{b}^{*} \mathbf{J}_{k} \mathbf{a}_{*}\right)=\sum_{k=1}^{K+1} \operatorname{Re}\left(\lambda_{k}^{*}\right)
$$

where $\mathbf{b}^{*}$ and $\mathbf{a}_{*}$ are the left and right eigenvectors, respectively, of the Jacobian $\mathbf{J}$ that relate to $\lambda^{*}$ and $\mathbf{J}_{k}$ is the contribution of each of the $K+1$ processes in $\mathbf{J}$; i.e., $\mathbf{J}=$ $\mathbf{J}_{1}+\mathbf{J}_{2}+\cdots+\mathbf{J}_{K+1}$. The quantity $B I^{k}$ in Eq. (13) can thus be cast as:

$$
B I^{k}=\frac{\operatorname{Re}\left(\mathbf{b}^{*} \mathbf{J}_{k} \mathbf{a}_{*}\right)}{\max _{k=1, K}\left|\operatorname{Re}\left(\mathbf{b}^{*} \mathbf{J}_{k} \mathbf{a}_{*}\right)\right|} \quad k=1, \ldots, K+1
$$


Considering the case of a real eigenvalue, a comparison of the expression for $B I^{k}$ with the equivalent CSP quantity $J_{k}^{i}$ in Eq. (8) reveals that $B I^{k}$ is a rescaled $J_{k}^{i}$. The same conclusion can be drawn in the case where the eigenvalue is complex [57]. This similarity is acknowledged in the $2014 \mathrm{C} \& \mathrm{~F}$ paper [40]. However, it is stated that "while the definition of BI is similar to that of the timescale importance index in CSP, the two concepts carry distinct physical meanings and differ in two major aspects." The first aspect is that "the timescale importance index was defined based on the timescales of CSP modes, while BI is defined for the specific mode leading to the limit phenomenon at a bifurcation point, where $\operatorname{Re}\left(\lambda_{*}\right)=0$ and the concept of timescale does not apply." This assertion is not correct because the eigenvalue with $\operatorname{Re}\left(\lambda_{*}\right)=0$ is one of those computed in the context of CSP. Moreover, the time scale - as introduced in 1989 - is the inverse of the eigenvalue modulus $[8,16]$, not simply as the inverse of its real part. The second aspect is that "BI is defined on eigenmodes that may involve complex eigenvalues and eigenvectors, while the timescale importance index in CSP was defined in the real space." This assertion is not correct either, since the the CSP time scale participation index was introduced in both the real and complex plane in the 2006 SIAM paper [57].

\section{Comparison of the two methods}

The previous discussion established the following regarding the various concepts and algorithmic tools introduced in the model reduction literature by CSP that were later incorporated in CEMA:

- The concept of the "mode" (reaction group) and of its "time scale", either explosive or dissipative (relaxation), was introduced by CSP in 1989 [8].

- The terms "explosive eigenvalue", "explosive time scale" and "explosive mode", were introduced by CSP in 1989 [8, 57, 70,72,76].

- The selection of the right and left eigenvectors of the Jacobian of the chemical kinetic source term as basis vectors and their duals, respectively, was introduced by CSP in $1989[8,18,19,21,23,70,72,73]$ and were used in all CSP investigations of the explosive mode [8, 22, 52-66].

- The "Explosion Pointer" introduced in 2009 and 2010 by CEMA [33, 36] is the same as the CSP (radical) Pointer, introduced in 1992 [48], while the "Explosion Index" $[34,36]$ is a rescaled $C S P$ (radical) Pointer.

- The "Participation Index" introduced in 2012 by CEMA [38] is similar to the CSP Participation Index (PI) introduced in 1990 and 1999 [69, 78]. The difference is that in CEMA the net reactions rate is considered, while in CSP the forward and backward directions are considered separately.

- The "Bifurcation Index" introduced in 2014 by CEMA [40] is a rescaled expression of the CSP Time scale Participation Index (TPI) introduced in 2006 [57]. 


\section{Conclusions}

There are currently two acronyms for identifying and analyzing explosive modes, using similar tools: CSP (first used in 1989 [8] and in more detail after 1997 [52, 54-56]) and CEMA (first used in 2009 [33, 34] and in more detail after 2010 [35-37]).

The comparative analysis of CSP and CEMA carried out here reveals that the theoretical foundation and the algorithmic tools employed in CEMA follow those already developed in the CSP framework. It clarifies the relationship between various CSP and CEMA definitions and provides useful guidelines to the community.

Recognition of the origin of the CSP algorithmic tools incorporated into CEMA is important in understanding the multi-scale context (fast/slow dynamics), which is essential in conducting an explosive mode analysis.

\section{References}

[1] F. A. Williams, Lectures on applied mathematics in combustion: Past contributions and future problems in laminar and turbulent combustion, Physica D: Nonlinear Phenomena 20 (1) (1986) 21-34.

[2] J. Buckmaster, The contribution of asymptotics to combustion, Physica D: Nonlinear Phenomena 20 (1) (1986) 91-108.

[3] J. Buckmaster, P. Clavin, A. Linan, M. Matalon, N. Peters, G. Sivashinsky, F. A. Williams, Combustion theory and modeling, Proceedings of the Combustion Institute 30 (1) (2005) 1-19.

[4] N. Peters, Numerical and asymptotic analyses of systematically reduced reaction schemes for hydrocarbon flames, in: B. L. R. Glowinski, R. Temam (Eds.), Numerical Simulation of Combustion Phenomena, Lecture Notes in Physics 241, Springer-Verlag, Berlin, 1985, pp. 90-109.

[5] N. Peters, R. J. Kee, The computation of stretched laminar methane-air diffusion flames using a reduced four-step mechanism, Combustion and Flame 68 (1) (1987) 17-29.

[6] N. Peters, F. A. Williams, The asymptotic structure of stoichiometric methane - air flames, Combustion and Flame 68 (2) (1987) 185-207.

[7] W. P. Jones, R. P. Lindstedt, Global reaction schemes for hydrocarbon combustion, Combustion and Flame 73 (3) (1988) 233-249.

[8] S. H. Lam, D. A. Goussis, Understanding complex chemical kinetics with Computational Singular Perturbation, Proceedings of the Combustion Institute 22 (1989) 931-941.

[9] H. G. Kaper, T. J. Kaper, Asymptotic analysis of two reduction methods for systems of chemical reactions, Physica D 165 (2002) 66-93.

[10] D. A. Goussis, M. Valorani, An efficient iterative algorithm for the approximation of the fast and slow dynamics of stiff systems, Journal of Computational Physics 214 (1) (2006) $316-346$. 
[11] D. A. Goussis, U. Maas, Model reduction for combustion chemistry, in: T. Echekki, E. Mastorakos (Eds.), Turbulent Combustion Modeling, Fluid Mechanics and its Applications, Springer London, 2011, pp. 193-220.

[12] T. Løvås, Model reduction techniques for chemical mechanisms, in: V. Patel (Ed.), Chemical Kinetics, InTech, Croatia, 2012, pp. 79-114.

[13] A. S. Tomlin, T. Turányi, Mechanism reduction to skeletal form and species lumping, in: F. Battin-Leclerc, J. M. Simmie, E. Blurock (Eds.), Cleaner Combustion, Green Energy and Technology, Springer, London, 2013, pp. 447-466.

[14] U. Maas, A. S. Tomlin, Time-scale splitting-based mechanism reduction, in: F. BattinLeclerc, J. M. Simmie, E. Blurock (Eds.), Cleaner Combustion, Green Energy and Technology, Springer London, 2013, pp. 467-484.

[15] T. Turányi, A. S. Tomlin, Analysis of Kinetic Reaction Mechanisms, Springer, Berlin, 2014.

[16] M. Valorani, D. A. Goussis, Explicit time-scale splitting algorithms for stiff problems: auto-ignition of gaseous mixtures behind a steady shock, J. Comp. Phys. 169 (2001) $44-79$.

[17] B. J. Debusschere, Y. M. Marzouk, H. N. Najm, B. Rhoads, D. A. Goussis, M. Valorani, Computational singular perturbation with non-parametric tabulation of slow manifolds for time integration of stiff chemical kinetics, Combustion Theory and Modelling 16 (1) (2012) 173-198.

[18] A. Massias, D. Diamantis, E. Mastorakos, D. A. Goussis, An algorithm for the construction of global reduced mechanisms with CSP data, Combustion and Flame 117 (4) (1999) 685-708.

[19] J. C. Lee, H. N. Najm, S. Lefantzi, J. Ray, M. Frenklach, M. Valorani, D. A. Goussis, A CSP and tabulation-based adaptive chemistry model, Combustion Theory and Modelling 11 (1) (2007) 73-102.

[20] M. Valorani, F. Creta, D. A. Goussis, J. C. Lee, H. N. Najm, An automatic procedure for the simplification of chemical kinetic mechanisms based on CSP, Combustion and Flame 146 (1-2) (2006) 29-51.

[21] M. Valorani, H. N. Najm, D. A. Goussis, CSP analysis of a transient flame-vortex interaction: Time scales and manifolds, Combustion and Flame 134 (1-2) (2003) 35-53.

[22] J. Prager, H. N. Najm, M. Valorani, D. A. Goussis, Structure of n-heptane/air triple flames in partially-premixed mixing layers, Combustion and Flame 158 (2011) 21282144 .

[23] A. Zagaris, H. G. Kaper, T. J. Kaper, Analysis of the computational singular perturbation reduction method for chemical kinetics, Journal of Nonlinear Science 19 (2004) $59-91$. 
[24] A. Zagaris, H. G. Kaper, T. J. Kaper, Fast and slow dynamics for the computational singular perturbation method, Multiscale Modeling \& Simulation 2 (4) (2004) 613-638.

[25] H. G. Kaper, T. J. Kaper, A. Zagaris, Geometry of the Computational Singular Perturbation method, Math. Model. Nat. Phenom. 10 (3) (2015) 16-30.

[26] I. Lizarraga, M. Wechselberger, Computational singular perturbation method for nonstandard slow-fast systems, SIAM Journal on Applied Dynamical Systems 19 (2) (2020) 994-1028.

[27] U. Maas, S. B. Pope, Implementation of simplified chemical kinetics based on intrinsic low-dimensional manifolds, Symposium (International) on Combustion 24 (1) (1992) $103-112$.

[28] U. Maas, S. B. Pope, Simplifying chemical kinetics: Intrinsic low-dimensional manifolds in composition space, Combustion and Flame 88 (3) (1992) 239 - 264.

[29] M. Valorani, S. Paolucci, The G-scheme: A framework for multi-scale adaptive model reduction, Journal of Computational Physics 228 (13) (2009) 4665 - 4701.

[30] M. Valorani, P. P. Ciottoli, R. M. Galassi, S. Paolucci, T. Grenga, E. Martelli, Enhancements of the G-scheme framework, Flow, Turbulence and Combustion 101 (4) (2018) $1023-1033$.

[31] M. Valorani, S. Paolucci, E. Martelli, T. Grenga, P. P. Ciottoli, Dynamical system analysis of ignition phenomena using the tangential stretching rate concept, Combustion and Flame 162 (8) (2015) 2963-2990.

[32] M. Valorani, P. P. Ciottoli, R. M. Galassi, Tangential stretching rate (TSR) analysis of non premixed reactive flows, Proceedings of the Combustion Institute 36 (1) (2017) $1357-1367$.

[33] T. Lu, C. Yoo, J. Chen, The role of chemical explosive mode in flames, in: Fall Technical Meeting, the Eastern Section Meeting of the Combustion Institute, College Park, USA, 2009.

[34] T. Lu, M. Wang, C. S. Yoo, J. H. Chen, C. K. Law, A mode tracking method for flow classification, in: 6th US National Combustion Meeting, Ann Arbor, Michigan, 2009.

[35] W. Liu, A. Kelley, C. K. Law, Flame propagation and counterflow nonpremixed ignition of mixtures of methane and ethylene, Combustion and Flame 157 (5) (2010) 1027-1036.

[36] T. F. Lu, C. S. Yoo, J. H. Chen, C. K. Law, Three-dimensional direct numerical simulation of a turbulent lifted hydrogen jet flame in heated coflow: a chemical explosive mode analysis, Journal of Fluid Mechanics 652 (2010) 45-64.

[37] J. H. Chen, Petascale direct numerical simulation of turbulent combustion - fundamental insights towards predictive models, Proceedings of the Combustion Institute 33 (1) (2011) $99-123$. 
[38] R. Shan, C. S. Yoo, J. H. Chen, T. Lu, Computational diagnostics for n-heptane flames with chemical explosive mode analysis, Combustion and Flame 159 (10) (2012) 31193127 .

[39] M. B. Luong, Z. Luo, T. Lu, S. H. Chung, C. S. Yoo, Direct numerical simulations of the ignition of lean primary reference fuel/air mixtures with temperature inhomogeneities, Combustion and Flame 160 (10) (2013) 2038 - 2047.

[40] R. Shan, T. Lu, A bifurcation analysis for limit flame phenomena of DME/air in perfectly stirred reactors, Combustion and Flame 161 (7) (2014) 1716-1723.

[41] C. Fureby, K. Nordin-Bates, K. Petterson, A. Bresson, V. Sabelnikov, A computational study of supersonic combustion in strut injector and hypermixer flow fields, Proceedings of the Combustion Institute 35 (2) (2015) 2127 - 2135.

[42] D. Han, S. Deng, W. Liang, P. Zhao, F. Wu, Z. Huang, C. K. Law, Laminar flame propagation and nonpremixed stagnation ignition of toluene and xylenes, Proceedings of the Combustion Institute 36 (1) (2017) 479 - 489.

[43] C. Xu, A. Y. Poludnenko, X. Zhao, H. Wang, T. Lu, Structure of strongly turbulent premixed n-dodecane-air flames: Direct numerical simulations and chemical explosive mode analysis, Combustion and Flame 209 (2019) 27 - 40.

[44] E.-A. Tingas, D. C. Kyritsis, D. A. Goussis, Comparative investigation of homogeneous autoignition of $\mathrm{DMe} /$ air and $\mathrm{EtOH} /$ air mixtures at low initial temperatures, Combustion Theory and Modelling 21 (1) (2017) 93-119.

[45] S. M. Sarathy, E.-A. Tingas, E. F. Nasir, A. Detogni, Z. Wang, A. Farooq, H. Im, Threestage heat release in n-heptane auto-ignition, Proceedings of the Combustion Institute 37 (1) (2019) $485-492$.

[46] E.-A. Tingas, Z. Wang, S. Mani Sarathy, H. G. Im, D. A. Goussis, Chemical kinetic insights into the ignition dynamics of n-hexane, Combustion and Flame 188 (2018) 28 -40 .

[47] E. A. Tingas, D. C. Kyritsis, D. A. Goussis, Autoignition dynamics of DMe/air and EtOH/air homogeneous mixtures, Combustion and Flame 162 (9) (2015) 3263 - 3276.

[48] D. A. Goussis, S. H. Lam, A study of homogeneous methanol oxidation kinetics using CSP, Symposium (International) on Combustion 24 (1) (1992) 113-120.

[49] S. H. Lam, D. A. Goussis, CSP method for simplifying kinetics, Int. J. Chem. Kinet. 26 (4) (1994) 461-486.

[50] D. M. Manias, E.-A. Tingas, F. E. Hernández Pérez, R. Malpica Galassi, P. Paolo Ciottoli, M. Valorani, H. G. Im, Investigation of the turbulent flame structure and topology at different karlovitz numbers using the tangential stretching rate index, Combustion and Flame 200 (2019) 155 - 167. 
[51] D. M. Manias, E.-A. Tingas, Y. Minamoto, H. G. Im, Topological and chemical characteristics of turbulent flames at mild conditions, Combustion and Flame 208 (2019) 86 98.

[52] C. Fotache, T. Kreutz, C. K. Law, Ignition of counterflowing methane versus heated air under reduced and elevated pressures, Combustion and Flame 108 (4) (1997) 442-470.

[53] J. C. Lee, H. N. Najm, S. Lefantzi, J. Ray, M. Frenklach, M. Valorani, D. A. Goussis, On chain branching and its role in homogeneous ignition and premixed flame propagation, Computational fluid and solid mechanics (2005) 717-720.

[54] A. Kazakov, M. Chaos, Z. Zhao, F. L. Dryer, Computational singular perturbation analysis of two-stage ignition of large hydrocarbons, The Journal of Physical Chemistry A 110 (21) (2006) 7003-7009.

[55] Z. Chen, X. Qin, Y. Ju, Z. Zhao, M. Chaos, F. L. Dryer, High temperature ignition and combustion enhancement by dimethyl ether addition to methane-air mixtures, Proceedings of the Combustion Institute 31 (1) (2007) 1215-1222.

[56] G. Mittal, M. Chaos, C.-J. Sung, F. L. Dryer, Dimethyl ether autoignition in a rapid compression machine: Experiments and chemical kinetic modeling, Fuel Processing Technology 89 (12) (2008) 1244-1254.

[57] D. A. Goussis, H. N. Najm, Model reduction and physical understanding of slowly oscillating processes: The circadian cycle, Multiscale Modeling \& Simulation 5 (4) (2006) $1297-1332$.

[58] T. Lu, C. Yoo, J. H. Chen, C. K. Law, Analysis of a turbulent lifted hydrogen/air jet flame from direct numerical simulation with computational singular perturbation, in: 46th AIAA Aerospace Sciences Meeting and Exhibit, 2008, p. 1013.

[59] D. J. Diamantis, D. C. Kyritsis, D. A. Goussis, The reactions favoring or opposing the development of explosive modes: auto-ignition of a homogeneous methane/air mixture, Proc. Combust. Inst. 35 (2015) 267-274.

[60] D. J. Diamantis, E. Mastorakos, D. A. Goussis, $\mathrm{H}_{2} /$ air autoignition: The nature and interaction of the developing explosive modes, Combust. Theor. Model. 19 (2015) 382433.

[61] E. A. Tingas, D. C. Kyritsis, D. A. Goussis, Autoignition dynamics of DME/air and EtOH/air homogeneous mixtures, Combust. Flame 162 (9) (2015) 3263-3276.

[62] E.-A. Tingas, D. C. Kyritsis, D. A. Goussis, Comparative investigation of homogeneous autoignition of DME/air and EtOH/air mixtures at low initial temperatures, Combust. Theor. Model. 21 (1) (2017) 93-119.

[63] E. A. Tingas, D. C. Kyritsis, D. A. Goussis, Algorithmic determination of the mechanism through which $\mathrm{H}_{2} \mathrm{O}$-dilution affects autoignition dynamics and $\mathrm{NO}$ formation in $\mathrm{CH}_{4}$ /air mixtures, Fuel 183 (2016) 90-98. 
[64] E.-A. Tingas, D. C. Kyritsis, D. A. Goussis, H2/air autoignition dynamics around the third explosion limit, Journal of Energy Engineering 145 (1) (2019) 04018074.

[65] E. Singh, E.-A. Tingas, D. A. Goussis, H. G. Im, S. M. Sarathy, Chemical ignition characteristics of ethanol blending with primary reference fuels, Energy \& Fuels 33 (10) (2019) 10185-10196.

[66] A. T. Khalil, D. M. Manias, D. C. Kyritsis, D. A. Goussis, NO formation and autoignition dynamics during combustion of H2O-diluted NH3/H2O2 mixtures with air, Energies 14 (1) (2020) 84.

[67] J. Liang, W. Jia, Y. Sun, Q.-D. Wang, Skeletal chemical kinetic mechanism generation for methanol combustion and systematic analysis on the ignition characteristics, AsiaPacific Journal of Chemical Engineering 15 (3) (2020) e2434.

[68] N. A. K. Doan, S. Bansude, K. Osawa, Y. Minamoto, T. Lu, J. H. Chen, N. Swaminathan, Identification of combustion mode under mild conditions using chemical explosive mode analysis, Proceedings of the Combustion Institute.

[69] D. A. Goussis, S. H. Lam, P. Gnoffo, Reduced and simplified chemical kinetics for air dissociation using computational singular perturbation, in: 28th Aerospace Sciences Meeting, 1990, p. 644.

[70] S. H. Lam, D. A. Goussis, Conventional asymptotics and computational singular perturbation for simplified kinetics modelling, in: Reduced kinetic mechanisms and asymptotic approximations for methane-air flames, Springer, 1991, pp. 227-242.

[71] M. Hadjinicolaou, D. A. Goussis, Asymptotic solution of stiff PDEs with the CSP method: the reaction diffusion equation, SIAM J. Sci. Comput. 20 (1998) 781-810.

[72] S. H. Lam, D. A. Goussis, Basic theory and demonstrations of computational singular perturbation for stiff equations, in: 12th IMACS World Congress on Scientific Computation, Paris, France, July, 1988, pp. 18-22.

[73] S. H. Lam, D. A. Goussis, D. Konopka, Time-resolved simplified chemical kinetics modelling using computational singular perturbation, in: 27th Aerospace Sciences Meeting, 1989, p. 575.

[74] S. H. Lam, Using CSP to understand complex chemical kinetics, Combustion Science and Technology 89 (5-6) (1993) 375-404.

[75] D. A. Goussis, M. Valorani, F. Creta, H. N. Najm, Reactive and reactive-diffusive time scales in stiff reaction-diffusion systems, Progress in Computational Fluid Dynamics, an International Journal 5 (6) (2005) 316-326.

[76] C. Trevino, F. Solorio, Asymptotic analysis of the high-temperature ignition of CO/H2/O2 mixtures, Combustion and Flame 86 (3) (1991) 285-295. 
[77] C. Trevino, Ignition phenomena in H2-O2 mixtures, Progress in Astronautics and Aeronautics, AIAA 131 (1991) 19-43.

[78] H. N. Najm, M. Valorani, D. A. Goussis, J. Prager, Analysis of methane-air edge flame structure, Combust. Theor. Model. 14 (2) (2010) 257-294. 